\title{
OPERATOR THEORY ON NONCOMMUTATIVE VARIETIES, II
}

\author{
GELU POPESCU
}

(Communicated by Joseph A. Ball)

\begin{abstract}
An $n$-tuple of operators $T:=\left[T_{1}, \ldots, T_{n}\right]$ on a Hilbert space $\mathcal{H}$ is called a $J$-constrained row contraction if $T_{1} T_{1}^{*}+\cdots+T_{n} T_{n}^{*} \leq I_{\mathcal{H}}$ and

$$
f\left(T_{1}, \ldots, T_{n}\right)=0, \quad f \in J,
$$

where $J$ is a WOT-closed two-sided ideal of the noncommutative analytic Toeplitz algebra $F_{n}^{\infty}$ and $f\left(T_{1}, \ldots, T_{n}\right)$ is defined using the $F_{n}^{\infty}$-functional calculus for row contractions.

We show that the constrained characteristic function $\Theta_{J, T}$ associated with $J$ and $T$ is a complete unitary invariant for $J$-constrained completely noncoisometric (c.n.c.) row contractions. We also provide a model for this class of row contractions in terms of the constrained characteristic functions. In particular, we obtain a model theory for $q$-commuting c.n.c. row contractions.
\end{abstract}

\section{INTRODUCTION}

In [22, we developed a dilation theory on noncommutative varieties determined by row contractions $T:=\left[T_{1}, \ldots, T_{n}\right]$ subject to constraints such as

$$
p\left(T_{1}, \ldots, T_{n}\right)=0, \quad p \in \mathcal{P},
$$

where $\mathcal{P}$ is a set of noncommutative polynomials. In this setting, the model $n$-tuple is the universal row contraction $\left[B_{1}, \ldots, B_{n}\right]$ satisfying the same constraints as $T$, which turns out to be the maximal constrained piece of the $n$-tuple $\left[S_{1}, \ldots, S_{n}\right]$ of left creation operators on the full Fock space on $n$ generators. We obtained a Beurling-type theorem characterizing the invariant subspaces under each operator $B_{1} \otimes I_{\mathcal{H}}, \ldots, B_{n} \otimes I_{\mathcal{H}}$, and Wold type decompositions for $*$-representations of the $C^{*}$-algebra $C^{*}\left(B_{1}, \ldots, B_{n}\right)$ generated by $B_{1}, \ldots, B_{n}$ and the identity. The constrained dilation and model theory is based on a class of constrained Poisson kernels associated with $T$ and representations of the Toeplitz algebra $C^{*}\left(B_{1}, \ldots, B_{n}\right)$.

Following the classical Sz.-Nagy-Foiaş model theory for a single contraction 23 and the multivariable noncommutative dilation theory $[8,9], 10,11,12$, we introduced in 22] a constrained characteristic function $\Theta_{J, T}$ associated with any constrained row contraction $T$. It turned out that, for constrained pure row contractions, the constrained characteristic function is a complete unitary invariant.

Received by the editors September 19, 2005 and, in revised form, March 20, 2006.

2000 Mathematics Subject Classification. Primary 47A20, 47A56; Secondary 47A13, 47A63.

Key words and phrases. Multivariable operator theory, noncommutative variety, characteristic function, model theory, row contraction, constrained shift, Poisson kernel, Fock space, unitary invariant, von Neumann inequality.

This research was supported in part by an NSF grant. 
We also showed that the curvature invariant and Euler characteristic asssociated with a Hilbert module generated by an arbitrary (resp. commuting) row contraction $T$ can be expressed only in terms of the (resp. constrained) characteristic function of $T$. This paper is a continuation of [22]. We further investigate the constrained characteristic function in several variables.

Let $H_{n}$ be an $n$-dimensional complex Hilbert space with orthonormal basis $e_{1}$, $e_{2}, \ldots, e_{n}$, where $n \in\{1,2, \ldots\}$. We consider the full Fock space of $H_{n}$ defined by

$$
F^{2}\left(H_{n}\right):=\bigoplus_{k \geq 0} H_{n}^{\otimes k}
$$

where $H_{n}^{\otimes 0}:=\mathbb{C} 1$ and $H_{n}^{\otimes k}$ is the (Hilbert) tensor product of $k$ copies of $H_{n}$. Define the left creation operators $S_{i}: F^{2}\left(H_{n}\right) \rightarrow F^{2}\left(H_{n}\right), i=1, \ldots, n$, by

$$
S_{i} \varphi:=e_{i} \otimes \varphi, \quad \varphi \in F^{2}\left(H_{n}\right) .
$$

The noncommutative analytic Toeplitz algebra $F_{n}^{\infty}$ and its norm closed version, the noncommutative disc algebra $\mathcal{A}_{n}$, were introduced by the author in [14] (see also [15]). $F_{n}^{\infty}$ is the algebra of left multipliers of the Fock space $F^{2}\left(H_{n}\right)$ and can be identified with the weakly closed (or $w^{*}$-closed) algebra generated by the left creation operators $S_{1}, \ldots, S_{n}$ and the identity. The noncommutative disc algebra $\mathcal{A}_{n}$ is the norm closed algebra generated by the same operators. When $n=1, F_{1}^{\infty}$ can be identified with $H^{\infty}(\mathbb{D})$, the algebra of bounded analytic functions on the open unit disc. The noncommutative analytic Toeplitz algebra $F_{n}^{\infty}$ can be viewed as a multivariable noncommutative analogue of $H^{\infty}(\mathbb{D})$.

Let $T:=\left[T_{1}, \ldots, T_{n}\right]$ be a completely noncoisometric (c.n.c.) row contraction (see Section 1 for notation) with $T_{i} \in B(\mathcal{H})$, the algebra of all bounded linear operators operators on a Hilbert space $\mathcal{H}$. Given a WOT-closed two-sided ideal $J$ of the noncommutative analytic Toeplitz algebra $F_{n}^{\infty}$, we say that $T$ is a $J$-constrained row contraction if

$$
f\left(T_{1}, \ldots, T_{n}\right)=0, \quad f \in J,
$$

where $f\left(T_{1}, \ldots, T_{n}\right)$ is defined using the $F_{n}^{\infty}$-functional calculus for c.n.c. row contractions [15]. In Section 1, we present some results concerning constrained Poisson transforms associated with $J$-constrained row contractions. More about noncommutative Poisson kernels and Poisson transforms on $C^{*}$-algebras generated by isometries can be found in [17, [2], [18, [19, [21, and 22.

The constrained characteristic function associated with a $J$-constrained row contraction $T$ was introduced in 22, as a multi-analytic operator $\Theta_{J, T}: \mathcal{N}_{J} \otimes \mathcal{D}_{T^{*}} \rightarrow$ $\mathcal{N}_{J} \otimes \mathcal{D}_{T}$ uniquely defined by the formal Fourier representation

$$
\begin{aligned}
-I_{\mathcal{N}_{J}} \otimes T+\left(I_{\mathcal{N}_{J}} \otimes \Delta_{T}\right)\left(I_{\mathcal{N}_{J} \otimes \mathcal{H}}-\right. & \left.\sum_{i=1}^{n} W_{i} \otimes T_{i}^{*}\right)^{-1} \\
& \times\left[W_{1} \otimes I_{\mathcal{H}}, \ldots, W_{n} \otimes I_{\mathcal{H}}\right]\left(I_{\mathcal{N}_{J}} \otimes \Delta_{T^{*}}\right),
\end{aligned}
$$

where $W_{1}, \ldots, W_{n}$ are the constrained right creation operators associated with $J$ (see Section 1 for notation).

In Section 2, we show that the constrained characteristic function is a complete unitary invariant for the class of constrained c.n.c. row contractions. We also provide a model for this class of row contractions in terms of the constrained characteristic functions. All the results of this paper apply, in particular, to c.n.c. row 
contractions subject to constraints such as $p\left(T_{1}, \ldots, T_{n}\right)=0, p \in \mathcal{P}$, where $\mathcal{P}$ is a set of noncommutative polynomials.

In particular, we obtain a model theory for commuting c.n.c. row contractions. The characteristic function of a commuting row contraction $T:=\left[T_{1}, \ldots, T_{n}\right]$ is the compression to the symmetric Fock space of the noncommutative characteristic function introduced in [12]. As shown in [22] (using [3]), it can be identified with the operator-valued analytic function on the open unit ball of $\mathbb{C}^{n}$, given by

$$
\begin{gathered}
\Theta_{J_{c}, T}(z):=-T+\Delta_{T}\left(I-z_{1} T_{1}^{*}-\cdots-z_{n} T_{n}^{*}\right)^{-1}\left[z_{1} I_{\mathcal{H}}, \ldots, z_{n} I_{\mathcal{H}}\right] \Delta_{T^{*}}, \\
z=\left(z_{1}, \ldots, z_{n}\right) \in \mathbb{B}_{n} .
\end{gathered}
$$

In this particular setting, the characteristic function was proved to be a complete unitary invariant for pure row contractions in [6] and, independently, by the author in [22]. We should mention that, in the commutative case, there is a model theory (see e.g. [1] and references therein) for operator tuples $T$ satisfying positivity conditions of type $\frac{1}{K}\left(T, T^{*}\right) \geq 0$, where $K$ is a reproducing kernel associated with certain domains in $\mathbb{C}^{n}$. It will be interesting to see if there is a "constrained-version" of all this work.

After the completion of this paper, we received a preprint from T. Bhattacharyya, J. Eschmeier, and J. Sarkar [7, and also noticed a very recent paper of C. Benhida, and D. Timotin 4. Both papers deal with the characteristic function of a commuting row contraction, and there is some overlap, in this particular setting, with Section 2 of our paper. However, our results concerning the model theory for constrained row contractions are more general, and the proofs are based on noncommutative dilation theory, rather then reproducing kernel Hilbert space techniques.

\section{Constrained Poisson kernels}

In this section, we present some results concerning the constrained Poisson kernels associated with completely noncoisometric row contractions. These results are needed in Section 2.

We need to recall from [13, [14, [15, [16], and [20] a few facts concerning multianalytic operators on Fock spaces. We denote by $\mathbb{F}_{n}^{+}$the unital free semigroup on $n$ generators $g_{1}, \ldots, g_{n}$, and the identity $g_{0}$. The length of $\alpha \in \mathbb{F}_{n}^{+}$is defined by $|\alpha|:=k$ if $\alpha=g_{i_{1}} g_{i_{2}} \cdots g_{i_{k}}$, and $|\alpha|:=0$ if $\alpha=g_{0}$. If $T_{1}, \ldots, T_{n} \in B(\mathcal{H})$, define $T_{\alpha}:=T_{i_{1}} T_{i_{2}} \cdots T_{i_{k}}$ if $\alpha=g_{i_{1}} g_{i_{2}} \cdots g_{i_{k}}$, and $T_{g_{0}}:=I_{\mathcal{H}}$. Similarly, we denote $e_{\alpha}:=e_{i_{1}} \otimes \cdots \otimes e_{i_{k}}$ and $e_{g_{0}}:=1$. We say that a bounded linear operator $M$ acting from $F^{2}\left(H_{n}\right) \otimes \mathcal{K}$ to $F^{2}\left(H_{n}\right) \otimes \mathcal{K}^{\prime}$ is multi-analytic if

$$
M\left(S_{i} \otimes I_{\mathcal{K}}\right)=\left(S_{i} \otimes I_{\mathcal{K}^{\prime}}\right) M \quad \text { for any } i=1, \ldots, n .
$$

We can associate with $M$ a unique formal Fourier expansion $M\left(R_{1}, \ldots, R_{n}\right):=$ $\sum_{\alpha \in \mathbb{F}_{n}^{+}} R_{\alpha} \otimes \theta_{(\alpha)}$, for some operators $\theta_{(\alpha)} \in B\left(\mathcal{K}, \mathcal{K}^{\prime}\right)$, where $R_{i}:=U^{*} S_{i} U, \quad i=$ $1, \ldots, n$, are the right creation operators on $F^{2}\left(H_{n}\right)$ and $U$ is the (flipping) unitary operator on $F^{2}\left(H_{n}\right)$ mapping $e_{i_{1}} \otimes e_{i_{2}} \otimes \cdots \otimes e_{i_{k}}$ into $e_{i_{k}} \otimes \cdots \otimes e_{i_{2}} \otimes e_{i_{1}}$. Since the operator $M$ acts like its Fourier representation on "polynomials", we will identify them for simplicity. The set of all multi-analytic operators in $B\left(F^{2}\left(H_{n}\right) \otimes \mathcal{K}, F^{2}\left(H_{n}\right) \otimes \mathcal{K}^{\prime}\right)$ coincides with $R_{n}^{\infty} \bar{\otimes} B\left(\mathcal{K}, \mathcal{K}^{\prime}\right)$, the WOT-closed operator space generated by the spatial tensor product, where $R_{n}^{\infty}=U^{*} F_{n}^{\infty} U$. A multi-analytic operator is called inner if it is an isometry, and outer if it has dense range. 
Now let $J \neq F_{n}^{\infty}$ be a WOT-closed two-sided ideal of the noncommutative analytic Toeplitz algebra $F_{n}^{\infty}$. Define the subspaces of the full Fock space $F^{2}\left(H_{n}\right)$ by setting

$$
\mathcal{M}_{J}:=\overline{J F^{2}\left(H_{n}\right)} \text { and } \quad \mathcal{N}_{J}:=F^{2}\left(H_{n}\right) \ominus \mathcal{M}_{J} .
$$

Define the constrained left (resp. right) creation operators by setting

$$
B_{i}:=\left.P_{\mathcal{N}_{J}} S_{i}\right|_{\mathcal{N}_{J}} \quad \text { and } \quad W_{i}:=\left.P_{\mathcal{N}_{J}} R_{i}\right|_{\mathcal{N}_{J}}, \quad i=1, \ldots, n .
$$

Let $\mathcal{W}\left(B_{1}, \ldots, B_{n}\right)$ be the $w^{*}$-closed algebra generated by $B_{1}, \ldots, B_{n}$ and the identity. We proved in [2] that

$$
\mathcal{W}\left(B_{1}, \ldots, B_{n}\right)=\left.P_{\mathcal{N}_{J}} F_{n}^{\infty}\right|_{\mathcal{N}_{J}}=\left\{f\left(B_{1}, \ldots, B_{n}\right): f\left(S_{1}, \ldots, S_{n}\right) \in F_{n}^{\infty}\right\},
$$

where, according to the $F_{n}^{\infty}$-functional calculus for c.n.c. row contractions [15],

$$
f\left(B_{1}, \ldots, B_{n}\right)=\text { SOT- } \lim _{r \rightarrow 1} f\left(r B_{1}, \ldots, r B_{n}\right) .
$$

Note that if $\varphi \in J$, then $\varphi\left(B_{1}, \ldots, B_{n}\right)=0$. An operator $M \in B\left(\mathcal{N}_{J} \otimes \mathcal{K}, \mathcal{N}_{J} \otimes \mathcal{K}^{\prime}\right)$ is called multi-analytic with respect to the constrained shifts $B_{1}, \ldots, B_{n}$ if

$$
M\left(B_{i} \otimes I_{\mathcal{K}}\right)=\left(B_{i} \otimes I_{\mathcal{K}^{\prime}}\right) M, \quad i=1, \ldots, n .
$$

If in addition $M$ is partially isometric, then we call it inner. If $M$ has dense range, it is called outer. We recall from [20] that the set of all multi-analytic operators with respect to $B_{1}, \ldots, B_{n}$ coincides with

$$
\mathcal{W}\left(W_{1}, \ldots, W_{n}\right) \bar{\otimes} B\left(\mathcal{K}, \mathcal{K}^{\prime}\right)=\left.P_{\mathcal{N}_{J} \otimes \mathcal{K}^{\prime}}\left[R_{n}^{\infty} \bar{\otimes} B\left(\mathcal{K}, \mathcal{K}^{\prime}\right)\right]\right|_{\mathcal{N}_{J} \otimes \mathcal{K}},
$$

and a similar result holds for the algebra $\mathcal{W}\left(B_{1}, \ldots, B_{n}\right)$.

Now, let us review (see [17]) some basic properties for noncommutative Poisson transforms associated with row contractions $T:=\left[T_{1}, \ldots, T_{n}\right], T_{i} \in B(\mathcal{H})$. For each $0<r \leq 1$, define the defect operator $\Delta_{T, r}:=\left(I-r^{2} T_{1} T_{1}^{*}-\cdots-r^{2} T_{n} T_{n}^{*}\right)^{1 / 2}$. The Poisson kernel associated with $T$ is the family of operators

$$
K_{T, r}: \mathcal{H} \rightarrow F^{2}\left(H_{n}\right) \otimes \overline{\Delta_{T, r} \mathcal{H}}, \quad 0<r \leq 1,
$$

defined by

$$
K_{T, r} h:=\sum_{k=0}^{\infty} \sum_{|\alpha|=k} e_{\alpha} \otimes r^{|\alpha|} \Delta_{T, r} T_{\alpha}^{*} h, \quad h \in \mathcal{H} .
$$

When $r=1$, we denote $\Delta_{T}:=\Delta_{T, 1}$ and $K_{T}:=K_{T, 1}$. The operators $K_{T, r}$ are isometries if $0<r<1$ and

$$
K_{T}^{*} K_{T}=I_{\mathcal{H}}-\text { SOT- } \lim _{k \rightarrow \infty} \sum_{|\alpha|=k} T_{\alpha} T_{\alpha}^{*} .
$$

This shows that $K_{T}$ is an isometry if and only if $T$ is a pure row contraction ([11]), i.e.,

$$
\text { SOT- } \lim _{k \rightarrow \infty} \sum_{|\alpha|=k} T_{\alpha} T_{\alpha}^{*}=0 .
$$

A key property of the Poisson kernel is that

$$
K_{T, r}\left(r^{|\alpha|} T_{\alpha}^{*}\right)=\left(S_{\alpha}^{*} \otimes I\right) K_{T, r} \quad \text { for all } 0<r \leq 1, \alpha \in \mathbb{F}_{n}^{+} .
$$


When $T$ is a completely noncoisometric (c.n.c.) row-contraction, i.e., there is no $h \in \mathcal{H}, h \neq 0$, such that

$$
\sum_{|\alpha|=k}\left\|T_{\alpha}^{*} h\right\|^{2}=\|h\|^{2} \quad \text { for all } k=1,2, \ldots,
$$

an $F_{n}^{\infty}$-functional calculus was developed in [15]. We showed that if $f=\sum_{\alpha \in \mathbb{F}_{n}^{+}} a_{\alpha} S_{\alpha}$ is in $F_{n}^{\infty}$, then

$$
\Gamma_{T}(f)=f\left(T_{1}, \ldots, T_{n}\right):=\text { SOT }-\lim _{r \rightarrow 1} \sum_{k=0}^{\infty} \sum_{|\alpha|=k} r^{|\alpha|} a_{\alpha} T_{\alpha}
$$

exists and $\Gamma_{T}: F_{n}^{\infty} \rightarrow B(\mathcal{H})$ is a WOT-continuous completely contractive homomorphism.

Let $J \neq F_{n}^{\infty}$ be a WOT-closed two-sided ideal of $F_{n}^{\infty}$, and let $T:=\left[T_{1}, \ldots, T_{n}\right]$, $T_{i} \in B(\mathcal{H})$, be a row contraction. The constrained Poisson kernel associated with $J$ and $T$ is the operator $K_{J, T}: \mathcal{H} \rightarrow \mathcal{N}_{J} \otimes \overline{\Delta_{T} \mathcal{H}}$ defined by $K_{J, T}:=\left(P_{\mathcal{N}_{J}} \otimes I_{\overline{\Delta_{T} \mathcal{H}}}\right) K_{T}$, where $K_{T}$ is the Poisson kernel defined by relation (1.1) (case $r=1$ ).

The following theorem provides extensions of some results from [21] for constrained pure row contractions.

Theorem 1.1. Let $J \neq F_{n}^{\infty}$ be a WOT-closed two-sided ideal of $F_{n}^{\infty}$ and let $T:=$ $\left[T_{1}, \ldots, T_{n}\right], T_{i} \in B(\mathcal{H})$, be a c.n.c. row contraction such that

$$
\varphi\left(T_{1}, \ldots, T_{n}\right)=0, \quad \varphi \in J .
$$

Then

$$
K_{J, T} f\left(T_{1}, \ldots, T_{n}\right)^{*}=\left(f\left(B_{1}, \ldots, B_{n}\right)^{*} \otimes I_{\overline{\Delta_{T} \mathcal{H}}}\right) K_{J, T}
$$

for any $f\left(B_{1}, \ldots, B_{n}\right) \in \mathcal{W}\left(B_{1}, \ldots, B_{n}\right)$, where $K_{J, T}$ is the constrained Poisson kernel associated with $J$ and $T$.

Proof. Due to relation (1.4) (case $r=1$ ) we have

$$
K_{T}^{*}\left(p\left(S_{1}, \ldots, S_{n}\right) \otimes I_{\mathcal{H}}\right)=p\left(T_{1}, \ldots, T_{n}\right) K_{T}^{*}
$$

for any polynomial $p\left(S_{1}, \ldots, S_{n}\right)$. According to [15], if

$$
f\left(S_{1}, \ldots, S_{n}\right):=\sum_{k=0}^{\infty} \sum_{|\alpha|=k} a_{\alpha} S_{\alpha}
$$

is in $F_{n}^{\infty}$, then, for any $0<r<1, f_{r}\left(S_{1}, \ldots, S_{n}\right):=\sum_{k=0}^{\infty} \sum_{|\alpha|=k} r^{|\alpha|} a_{\alpha} S_{\alpha}$ is in the noncommutative disc algebra $\mathcal{A}_{n}$. Since

$$
\lim _{m \rightarrow \infty} \sum_{k=0}^{m} \sum_{|\alpha|=k} r^{|\alpha|} a_{\alpha} S_{\alpha}=f_{r}\left(S_{1}, \ldots, S_{n}\right)
$$

in the norm topology, relation (1.7) implies

$$
K_{T}^{*}\left(f_{r}\left(S_{1}, \ldots, S_{n}\right) \otimes I_{\mathcal{H}}\right)=f_{r}\left(T_{1}, \ldots, T_{n}\right) K_{T}^{*}
$$

for any $f\left(S_{1}, \ldots, S_{n}\right) \in F_{n}^{\infty}$ and $0<r<1$. Since $T$ is a c.n.c. row contraction and $S:=\left[S_{1}, \ldots, S_{n}\right]$ is a pure row contraction, we can use the $F_{n}^{\infty}$-functional calculus. We recall that the map $A \mapsto A \otimes I$ is SOT-continuous on bounded sets of $B\left(F^{2}\left(H_{n}\right)\right)$ and, due to the noncommutative von Neumann inequality [14] (see 
24 for the classical case), we have $\left\|f_{r}\left(S_{1}, \ldots, S_{n}\right)\right\| \leq\left\|f\left(S_{1}, \ldots, S_{n}\right)\right\|$. Therefore, we can take $r \rightarrow 1$ in the above equality and obtain

$$
K_{T}^{*}\left(f\left(S_{1}, \ldots, S_{n}\right) \otimes I_{\overline{\Delta_{T} \mathcal{H}}}\right)=f\left(T_{1}, \ldots, T_{n}\right) K_{T}^{*}
$$

for any $f\left(S_{1}, \ldots, S_{n}\right) \in F_{n}^{\infty}$, where $f\left(T_{1}, \ldots, T_{n}\right)$ is defined by formula (1.6).

Now, according to relation (1.8), we have

$$
\left\langle\left(\varphi\left(S_{1}, \ldots, S_{n}\right)^{*} \otimes I\right) K_{T} h, 1 \otimes k\right\rangle=\left\langle K_{T} \varphi\left(T_{1}, \ldots, T_{n}\right)^{*} h, 1 \otimes k\right\rangle
$$

for any $\varphi\left(S_{1}, \ldots, S_{n}\right) \in F_{n}^{\infty}, h \in \mathcal{H}$, and $k \in \overline{\Delta_{T} \mathcal{H}}$. Note that if $\varphi\left(S_{1}, \ldots, S_{n}\right) \in J$, then $\varphi\left(T_{1}, \ldots, T_{n}\right)=0$, and relation (1.9) implies $\left\langle K_{T} h, \varphi \otimes k\right\rangle=0$ for any $h, k \in$ $\mathcal{H}$. Taking into account the definition of $\mathcal{M}_{J}$, we deduce that

$$
K_{T}(\mathcal{H}) \subseteq \mathcal{N}_{J} \otimes \mathcal{H}
$$

This shows that the constrained Poisson kernel satisfies the relation

$$
K_{J, T} h=\left(P_{\mathcal{N}_{J}} \otimes I_{\overline{\Delta_{T} \mathcal{H}}}\right) K_{T} h=K_{T} h, \quad h \in \mathcal{H} .
$$

Since $J$ is a left ideal of $F_{n}^{\infty}, \mathcal{N}_{J}$ is an invariant subspace under each operator $S_{1}^{*}, \ldots, S_{n}^{*}$ and therefore $B_{\alpha}=P_{\mathcal{N}_{J}} S_{\alpha} \mid \mathcal{N}_{J}, \alpha \in \mathbb{F}_{n}^{+}$. Since $\left[B_{1}, \ldots, B_{n}\right]$ is a pure row contraction, we can use the $F_{n}^{\infty}$-functional calculus to deduce that

$$
f\left(B_{1}, \ldots, B_{n}\right)=P_{\mathcal{N}_{J}} f\left(S_{1}, \ldots, S_{n}\right) \mid \mathcal{N}_{J}
$$

for any $f\left(S_{1}, \ldots, S_{n}\right) \in F_{n}^{\infty}$. Taking into account relations (1.8), (1.11), and (1.12), we obtain

$$
\begin{aligned}
K_{J, T} f\left(T_{1}, \ldots, T_{n}\right)^{*} & =\left(P_{\mathcal{N}_{J}} \otimes I_{\overline{\Delta_{T} \mathcal{H}}}\right)\left[f\left(S_{1}, \ldots, S_{n}\right)^{*} \otimes I_{\overline{\Delta_{T} \mathcal{H}}}\right]\left(P_{\mathcal{N}_{J}} \otimes I_{\overline{\Delta_{T} \mathcal{H}}}\right) K_{T} \\
& =\left[\left(P_{\mathcal{N}_{J}} f\left(S_{1}, \ldots, S_{n}\right) \mid \mathcal{N}_{J}\right)^{*} \otimes I_{\overline{\Delta_{T} \mathcal{H}}}\right] K_{J, T} \\
& =\left[f\left(B_{1}, \ldots, B_{n}\right)^{*} \otimes I_{\overline{\Delta_{T} \mathcal{H}}}\right] K_{J, T} .
\end{aligned}
$$

Therefore, we have

$$
K_{J, T} f\left(T_{1}, \ldots, T_{n}\right)^{*}=\left[f\left(B_{1}, \ldots, B_{n}\right)^{*} \otimes I_{\overline{\Delta_{T} \mathcal{H}}}\right] K_{J, T}
$$

for any $f\left(B_{1}, \ldots, B_{n}\right) \in \mathcal{W}\left(B_{1}, \ldots, B_{n}\right)$. This completes the proof.

Corollary 1.2. Let $J \neq F_{n}^{\infty}$ be a WOT-closed two-sided ideal of $F_{n}^{\infty}$ and let $T:=\left[T_{1}, \ldots, T_{n}\right], T_{i} \in B(\mathcal{H})$, be a c.n.c. row contraction such that

$$
\varphi\left(T_{1}, \ldots, T_{n}\right)=0, \quad \varphi \in J .
$$

Then

$$
K_{J, T} T_{i}^{*}=\left(B_{i}^{*} \otimes I_{\overline{\Delta_{T} \mathcal{H}}}\right) K_{J, T}, \quad i=1, \ldots, n,
$$

and

$$
K_{J, T}^{*} K_{J, T}=I_{\mathcal{H}}-\text { SOT- } \lim _{k \rightarrow \infty} \sum_{|\alpha|=k} T_{\alpha} T_{\alpha}^{*},
$$

where $K_{J, T}$ is the constrained Poisson kernel associated with $T$ and $J$.

Moreover, if a map $\Psi: \mathcal{W}\left(B_{1}, \ldots, B_{n}\right) \rightarrow B(\mathcal{H})$ satisfies the relation

$$
\Psi(f) K_{J, T}^{*}=K_{J, T}^{*}(f \otimes I), \quad f \in \mathcal{W}\left(B_{1}, \ldots, B_{n}\right),
$$

then

$$
\Psi(f)=f\left(T_{1}, \ldots, T_{n}\right), \quad f \in \mathcal{W}\left(B_{1}, \ldots, B_{n}\right)
$$


Proof. The first part of the theorem follows easily from Theorem 1.1. Relation (1.14) is a consequence of (1.2) and (1.10). Due to relation (1.14), if $T$ is a c.n.c. row contraction, then $K_{J, T}$ is a one-to-one operator. Consequently, Theorem 1.1 and relation (1.15) imply $\Psi(f)=f\left(T_{1}, \ldots, T_{n}\right), f \in \mathcal{W}\left(B_{1}, \ldots, B_{n}\right)$. This completes the proof.

We remark that if $\mathcal{P}$ is a family of noncommutative polynomials in $S_{1}, \ldots, S_{n}$ and $T:=\left[T_{1}, \ldots, T_{n}\right]$ is an arbitrary row contraction such that $p\left(T_{1}, \ldots, T_{n}\right)=0$, $p \in \mathcal{P}$, then one can prove that $T$ is a c.n.c. row contraction if and only if $K_{J, T}$ is a one-to-one operator, where $J$ is the WOT-closed two-sided ideal of $F_{n}^{\infty}$ generated by $\mathcal{P}$.

\section{CONSTRAINED CHARACTERISTIC FUnCTIONS}

In this section, we show that the constrained characteristic function is a complete unitary invariant for the class of constrained c.n.c. row contractions. We also provide a model for this class of row contractions in terms of the constrained characteristic functions. All the results of this section apply, in particular, to c.n.c. row contraction subject to constraints such as

$$
p\left(T_{1}, \ldots, T_{n}\right)=0, \quad p \in \mathcal{P},
$$

where $\mathcal{P}$ is a set of noncommutative polynomials.

The characteristic function associated with an arbitrary row contraction $T:=$ $\left[T_{1}, \ldots, T_{n}\right], T_{i} \in B(\mathcal{H})$, was introduced in [12] (see [23] for the classical case $n=1$ ), and it was proved to be a complete unitary invariant for completely noncoisometric (c.n.c.) row contractions. Using the characterization of multi-analytic operators on Fock spaces (see [16], 18]), one can easily see that the characteristic function of $T$ is a multi-analytic operator

$$
\Theta_{T}: F^{2}\left(H_{n}\right) \otimes \mathcal{D}_{T^{*}} \rightarrow F^{2}\left(H_{n}\right) \otimes \mathcal{D}_{T}
$$

with the formal Fourier representation

$$
\begin{aligned}
-I_{F^{2}\left(H_{n}\right)} \otimes T+\left(I_{F^{2}\left(H_{n}\right)} \otimes \Delta_{T}\right) & \left(I_{F^{2}\left(H_{n}\right) \otimes \mathcal{H}}-\sum_{i=1}^{n} R_{i} \otimes T_{i}^{*}\right)^{-1} \\
& \times\left[R_{1} \otimes I_{\mathcal{H}}, \ldots, R_{n} \otimes I_{\mathcal{H}}\right]\left(I_{F^{2}\left(H_{n}\right)} \otimes \Delta_{T^{*}}\right),
\end{aligned}
$$

where $R_{1}, \ldots, R_{n}$ are the right creation operators on the full Fock space $F^{2}\left(H_{n}\right)$. Here, we need to clarify some notation since some of them are different from those considered in [12. The defect operators associated with a row contraction $T:=$ $\left[T_{1}, \ldots, T_{n}\right]$ are

$$
\Delta_{T}:=\left(I_{\mathcal{H}}-\sum_{i=1}^{n} T_{i} T_{i}^{*}\right)^{1 / 2} \in B(\mathcal{H}) \quad \text { and } \quad \Delta_{T^{*}}:=\left(I-T^{*} T\right)^{1 / 2} \in B\left(\mathcal{H}^{(n)}\right),
$$

while the defect spaces are $\mathcal{D}_{T}:=\overline{\Delta_{T} \mathcal{H}}$ and $\mathcal{D}_{T^{*}}:=\overline{\Delta_{T^{*}} \mathcal{H}^{(n)}}$, where $\mathcal{H}^{(n)}$ denotes the direct sum of $n$ copies of $\mathcal{H}$. We proved 22] that

$$
I_{F^{2}\left(H_{n}\right) \otimes \mathcal{D}_{T}}-\Theta_{T} \Theta_{T}^{*}=K_{T} K_{T}^{*},
$$

where $K_{T}$ is the Poisson kernel associated with $T$. 
Let $J \neq F_{n}^{\infty}$ be a WOT-closed two-sided ideal of the noncommutative analytic Toeplitz algebra $F_{n}^{\infty}$. In [22, we defined the constrained characteristic function associated with a $J$-constrained c.n.c. row contraction $T:=\left[T_{1}, \ldots, T_{n}\right], \quad T_{i} \in$ $B(\mathcal{H})$, to be the multi-analytic operator (with respect to the constrained shifts $\left.B_{1}, \ldots, B_{n}\right)$

$$
\Theta_{J, T}: \mathcal{N}_{J} \otimes \mathcal{D}_{T^{*}} \rightarrow \mathcal{N}_{J} \otimes \mathcal{D}_{T}
$$

defined by the formal Fourier representation

$$
\begin{aligned}
-I_{\mathcal{N}_{J}} \otimes T+\left(I_{\mathcal{N}_{J}} \otimes \Delta_{T}\right)\left(I_{\mathcal{N}_{J} \otimes \mathcal{H}}\right. & \left.-\sum_{i=1}^{n} W_{i} \otimes T_{i}^{*}\right)^{-1} \\
& \times\left[W_{1} \otimes I_{\mathcal{H}}, \ldots, W_{n} \otimes I_{\mathcal{H}}\right]\left(I_{\mathcal{N}_{J}} \otimes \Delta_{T^{*}}\right) .
\end{aligned}
$$

Taking into account that $\mathcal{N}_{J}$ is a co-invariant subspace under $R_{1}, \ldots, R_{n}$, we have

$$
\begin{aligned}
\Theta_{T}^{*}\left(\mathcal{N}_{J} \otimes \mathcal{D}_{T}\right) & \subseteq \mathcal{N}_{J} \otimes \mathcal{D}_{T^{*}} \text { and } \\
P_{\mathcal{N}_{J} \otimes \mathcal{D}_{T}} \Theta_{T} \mid \mathcal{N}_{J} \otimes \mathcal{D}_{T^{*}} & =\Theta_{J, T} .
\end{aligned}
$$

Let us remark that the above definition of the constrained characteristic function makes sense when $T:=\left[T_{1}, \ldots, T_{n}\right]$ is an arbitrary $J$-constrained row contraction and $J$ is a WOT-closed two-sided ideal of $F_{n}^{\infty}$ generated by a family of polynomials (see $[22]$ ).

The next result was obtained in [22] for a WOT-closed two-sided ideal of $F_{n}^{\infty}$ generated by polynomials. Here, we have an extension of that result.

Theorem 2.1. Let $J \neq F_{n}^{\infty}$ be a WOT-closed two-sided ideal of $F_{n}^{\infty}$ and let $T:=$ $\left[T_{1}, \ldots, T_{n}\right], T_{i} \in B(\mathcal{H})$, be a J-constrained c.n.c. row contraction. Then

$$
I_{\mathcal{N}_{J} \otimes \mathcal{D}_{T}}-\Theta_{J, T} \Theta_{J, T}^{*}=K_{J, T} K_{J, T}^{*},
$$

where $\Theta_{J, T}$ is the constrained characteristic function of $T$ and $K_{J, T}$ is the corresponding constrained Poisson kernel.

Proof. Due to relation (1.10), we have range $K_{T} \subseteq \mathcal{N}_{J} \otimes \overline{\Delta_{T} \mathcal{H}}$. Taking the compression of relation (2.1) to the subspace $\mathcal{N}_{J} \otimes \mathcal{D}_{T} \subset F^{2}\left(H_{n}\right) \otimes \mathcal{D}_{T}$, we obtain

$$
I_{\mathcal{N}_{J} \otimes \mathcal{D}_{T}}-P_{\mathcal{N}_{J} \otimes \mathcal{D}_{T}} \Theta_{T} \Theta_{T}^{*}\left|\mathcal{N}_{J} \otimes \mathcal{D}_{T}=P_{\mathcal{N}_{J} \otimes \mathcal{D}_{T}} K_{T} K_{T}^{*}\right| \mathcal{N}_{J} \otimes \mathcal{D}_{T} .
$$

Using relation (2.2) and that $W_{i}^{*}=R_{i}^{*} \mid \mathcal{N}_{J}, \quad i=1, \ldots, n$, we deduce (2.3). The proof is complete.

Now, we present a model for constrained c.n.c. row contractions in terms of the constrained characteristic functions. We recall that two row contractions $T:=$ $\left[T_{1}, \ldots, T_{n}\right], T_{i} \in B(\mathcal{H})$, and $T^{\prime}=:\left[T_{1}^{\prime}, \ldots, T_{n}^{\prime}\right], T_{i}^{\prime} \in B\left(\mathcal{H}^{\prime}\right)$, are equivalent if there is a unitary operator $U: \mathcal{H} \rightarrow \mathcal{H}^{\prime}$ such that $U T_{i}=T_{i}^{\prime} U$ for any $i=1, \ldots, n$.

Theorem 2.2. Let $J \neq F_{n}^{\infty}$ be a WOT-closed two-sided ideal of $F_{n}^{\infty}$ and let $T:=$ $\left[T_{1}, \ldots, T_{n}\right]$ be a c.n.c. row contraction such that

$$
\varphi\left(T_{1}, \ldots, T_{n}\right)=0, \quad \varphi \in J
$$

Then $T:=\left[T_{1}, \ldots, T_{n}\right]$ is unitarily equivalent to the constrained row contraction $\mathbb{T}:=\left[\mathbb{T}_{1}, \ldots, \mathbb{T}_{n}\right]$ on the Hilbert space

$$
\mathbb{H}_{J, T}:=\left[\left(\mathcal{N}_{J} \otimes \mathcal{D}_{T}\right) \oplus \overline{\Delta_{J, T}\left(\mathcal{N}_{J} \otimes \mathcal{D}_{T^{*}}\right)}\right] \ominus\left\{\Theta_{J, T} f \oplus \Delta_{J, T} f: f \in \mathcal{N}_{J} \otimes \mathcal{D}_{T^{*}}\right\}
$$


where $\Delta_{J, T}:=\left(I-\Theta_{J, T}^{*} \Theta_{J, T}\right)^{1 / 2}$ and each operator $\mathbb{T}_{i}, \quad i=1, \ldots, n$, is uniquely defined by the relation

$$
\left(\left.P_{\mathcal{N}_{J} \otimes \mathcal{D}_{T}}\right|_{\mathbb{H}_{J, T}}\right) \mathbb{T}_{i}^{*} x=\left(B_{i}^{*} \otimes I_{\mathcal{D}_{T}}\right)\left(\left.P_{\mathcal{N}_{J} \otimes \mathcal{D}_{T}}\right|_{\mathbb{H}_{J, T}}\right) x, \quad x \in \mathbb{H}_{J, T},
$$

where $\left.P_{\mathcal{N}_{J} \otimes \mathcal{D}_{T}}\right|_{\mathbb{H}_{J, T}}$ is a one-to-one operator, $P_{\mathcal{N}_{J} \otimes \mathcal{D}_{T}}$ is the orthogonal projection of the Hilbert space $\left(\mathcal{N}_{J} \otimes \mathcal{D}_{T}\right) \oplus \overline{\Delta_{J, T}\left(\mathcal{N}_{J} \otimes \mathcal{D}_{T^{*}}\right)}$ onto the subspace $\mathcal{N}_{J} \otimes \mathcal{D}_{T}$, and $B_{1}, \ldots, B_{n}$ are the constrained left creation operators determined by $J$.

Moreover, $T$ is a constrained pure row contraction if and only if the constrained characteristic function $\Theta_{J, T}$ is an inner multi-analytic operator with respect to $B_{1}, \ldots, B_{n}$. In this case, $T$ is unitarily equivalent to the row contraction

$$
\left[P_{\mathbb{H}_{J, T}}\left(B_{1} \otimes I_{\mathcal{D}_{T}}\right)\left|\mathbb{H}_{J, T}, \ldots, P_{\mathbb{H}_{J, T}}\left(B_{n} \otimes I_{\mathcal{D}_{T}}\right)\right| \mathbb{H}_{J, T}\right],
$$

where $P_{\mathbb{H}_{J, T}}$ is the orthogonal projection of $\mathcal{N}_{J} \otimes \mathcal{D}_{T}$ onto the Hilbert space

$$
\mathbb{H}_{J, T}:=\left(\mathcal{N}_{J} \otimes \mathcal{D}_{T}\right) \ominus \Theta_{J, T}\left(\mathcal{N}_{J} \otimes \mathcal{D}_{T^{*}}\right) .
$$

Proof. Consider the Hilbert space $\mathbb{K}_{J, T}:=\left(\mathcal{N}_{J} \otimes \mathcal{D}_{T}\right) \oplus \overline{\Delta_{J, T}\left(\mathcal{N}_{J} \otimes \mathcal{D}_{T^{*}}\right)}$ and define the operator $\Phi: \mathcal{N}_{J} \otimes \mathcal{D}_{T^{*}} \rightarrow \mathbb{K}_{J, T}$ by setting

$$
\Phi f:=\Theta_{J, T} f \oplus \Delta_{J, T} f, \quad f \in \mathcal{N}_{J} \otimes \mathcal{D}_{T^{*}} .
$$

Notice that $\Phi$ is an isometry and

$$
\Phi^{*}(g \oplus 0)=\Theta_{J, T}^{*} g, \quad g \in \mathcal{N}_{J} \otimes \mathcal{D}_{T} .
$$

Consequently, denoting by $P_{\mathbb{H}_{J, T}}$ the orthogonal projection of $\mathbb{K}_{J, T}$ onto the subspace $\mathbb{H}_{J, T}$, we have

$$
\begin{aligned}
\|g\|^{2} & =\left\|P_{\mathbb{H}_{J, T}}(g \oplus 0)\right\|^{2}+\left\|\Phi \Phi^{*}(g \oplus 0)\right\|^{2} \\
& =\left\|P_{\mathbb{H}_{J, T}}(g \oplus 0)\right\|^{2}+\left\|\Theta_{J, T}^{*} g\right\|^{2}
\end{aligned}
$$

for any $g \in \mathcal{N}_{J} \otimes \mathcal{D}_{T}$. On the other hand, due to Theorem 2.1

$$
\left\|K_{J, T}^{*} g\right\|^{2}+\left\|\Theta_{J, T}^{*} g\right\|^{2}=\|g\|^{2}, \quad g \in \mathcal{N}_{J} \otimes \mathcal{D}_{T} .
$$

Combining the above relations, we deduce that

$$
\left\|K_{J, T}^{*} g\right\|=\left\|P_{\mathbb{H}_{J, T}}(g \oplus 0)\right\|, \quad g \in \mathcal{N}_{J} \otimes \mathcal{D}_{T} .
$$

Since $\left[T_{1}, \ldots, T_{n}\right]$ is a constrained c.n.c. row contraction, Corollary 1.2 shows that $K_{J, T}$ is a one-to-one operator and range $K_{J, T}^{*}$ is dense in $\mathcal{H}$.

Let $x \in \mathbb{H}_{J, T}$ and assume that $x \perp P_{\mathbb{H}_{J, T}}(g \oplus 0)$ for any $g \in \mathcal{N}_{J} \otimes \mathcal{D}_{T}$. Using the definition of $\mathbb{H}_{J, T}$ and the fact that

$$
\mathbb{K}_{J, T}=\left\{g \oplus 0: g \in \mathcal{N}_{J} \otimes \mathcal{D}_{T}\right\} \bigvee\left\{\Theta_{J, T} f \oplus \Delta_{J, T} f, \quad f \in \mathcal{N}_{J} \otimes \mathcal{D}_{T^{*}}\right\}
$$

we deduce that $x=0$. This shows that

$$
\mathbb{H}_{J, T}=\left\{P_{\mathbb{H}_{J, T}}(g \oplus 0): g \in \mathcal{N}_{J} \otimes \mathcal{D}_{T}\right\}^{-} .
$$

Hence, and due to relation (2.6), there is a unique unitary operator $\Gamma: \mathcal{H} \rightarrow \mathbb{H}_{J, T}$ such that

$$
\Gamma\left(K_{J, T}^{*} g\right)=P_{\mathbb{H}_{J, T}}(g \oplus 0), \quad g \in \mathcal{N}_{J} \otimes \mathcal{D}_{T}
$$


Using Theorem 2.1. relation (2.5), and the fact that $\Phi$ is an isometry, we have

$$
\begin{aligned}
P_{\mathcal{N}_{J} \otimes \mathcal{D}_{T}} \Gamma K_{J, T}^{*} g & =P_{\mathcal{N}_{J} \otimes \mathcal{D}_{T}} P_{\mathbb{H}_{J}, T}(g \oplus 0) \\
& =g-P_{\mathcal{N}_{J} \otimes \mathcal{D}_{T}} \Phi \Phi^{*}(g \oplus 0) \\
& =g-\Theta_{J, T} \Theta_{J, T}^{*} g \\
& =K_{J, T} K_{J, T}^{*} g
\end{aligned}
$$

for any $g \in \mathcal{N}_{J} \otimes \mathcal{D}_{T}$. Consequently, since the range of $K_{J, T}^{*}$ is dense in $\mathcal{H}$, we deduce that

$$
P_{\mathcal{N}_{J} \otimes \mathcal{D}_{T}} \Gamma=K_{J, T}
$$

For each $i=1, \ldots, n$, let $\mathbb{T}_{i}: \mathbb{H}_{J, T} \rightarrow \mathbb{H}_{J, T}$ be defined by $\mathbb{T}_{i}:=\Gamma T_{i} \Gamma^{*}, i=$ $1, \ldots, n$. Due to relation (2.8) and taking into account that the constrained Poisson kernel $K_{J, T}$ is one-to-one, we deduce that

$$
\left.P_{\mathcal{N}_{J} \otimes \mathcal{D}_{T}}\right|_{\mathbb{H}_{J, T}}=K_{J, T} \Gamma^{*}
$$

is a one-to-one operator acting from $\mathbb{H}_{J, T}$ to $\mathcal{N}_{J} \otimes \mathcal{D}_{T}$. Notice also that, using relation (2.9) and Corollary 1.2 we have

$$
\begin{aligned}
\left(\left.P_{\mathcal{N}_{J} \otimes \mathcal{D}_{T}}\right|_{\mathbb{H}_{J, T}}\right) \mathbb{T}_{i}^{*} \Gamma h & =\left(\left.P_{\mathcal{N}_{J} \otimes \mathcal{D}_{T}}\right|_{\mathbb{H}_{J, T}}\right) \Gamma T_{i}^{*} h=K_{J, T} T_{i}^{*} h \\
& =\left(B_{i}^{*} \otimes I_{\mathcal{D}_{T}}\right) K_{J, T} h \\
& =\left(B_{i}^{*} \otimes I_{\mathcal{D}_{T}}\right)\left(\left.P_{\mathcal{N}_{J} \otimes \mathcal{D}_{T}}\right|_{\mathbb{H}_{J, T}}\right) \Gamma h
\end{aligned}
$$

for any $h \in \mathcal{H}$. Hence, we deduce that

$$
\left(\left.P_{\mathcal{N}_{J} \otimes \mathcal{D}_{T}}\right|_{\mathbb{H}_{J, T}}\right) \mathbb{T}_{i}^{*} x=\left(B_{i}^{*} \otimes I_{\mathcal{D}_{T}}\right)\left(\left.P_{\mathcal{N}_{J} \otimes \mathcal{D}_{T}}\right|_{\mathbb{H}_{J, T}}\right) x, \quad x \in \mathbb{H}_{J, T}
$$

Since the operator $\left.P_{\mathcal{N}_{J} \otimes \mathcal{D}_{T}}\right|_{\mathbb{H}_{J, T}}$ is one-to-one (see (2.9)), relation (2.10) uniquely determines the operators $\mathbb{T}_{i}^{*}, i=1, \ldots, n$.

To prove that last part of the theorem, assume that $T:=\left[T_{1}, \ldots, T_{n}\right]$ is a constrained pure row contraction. According to Corollary 1.2, the constrained Poisson kernel $K_{J, T}: \mathcal{H} \rightarrow \mathcal{N}_{J} \otimes \mathcal{D}_{T}$ is an isometry. Consequently, $K_{J, T} K_{J, T}^{*}$ is the orthogonal projection of $\mathcal{N}_{J} \otimes \mathcal{D}_{T}$ onto $K_{J, T} \mathcal{H}$. According to Theorem 2.1, relation (2.3) shows that $K_{J, T} K_{J, T}^{*}$ and $\Theta_{J, T} \Theta_{J, T}^{*}$ are mutually orthogonal projections such that

$$
K_{J, T} K_{J, T}^{*}+\Theta_{J, T} \Theta_{J, T}^{*}=I_{\mathcal{N}_{J} \otimes \mathcal{D}_{T}} .
$$

Therefore, $\Theta_{J, T}$ is a partial isometry, i.e., an inner multi-analytic operator, and $\Theta_{J, T}^{*} \Theta_{J, T}$ is a projection. This implies that $\Delta_{J, T}$ is the projection on the orthogonal complement of range $\Theta_{J, T}^{*}$.

Now, notice that a vector $u \oplus v \in \mathbb{K}_{J, T}$ is in $\mathbb{H}_{J, T}$ if and only if

$$
\left\langle u \oplus v, \Theta_{J, T} f \oplus \Delta_{J, T} f\right\rangle=0 \quad \text { for any } f \in \mathcal{N}_{J} \otimes \mathcal{D}_{T^{*}} .
$$

This is equivalent to

$$
\Theta_{J, T}^{*} u+\Delta_{J, T} v=0 .
$$

Due to the above observations, we have $\Theta_{J, T}^{*} u \perp \Delta_{J, T} v$. This shows that relation (2.11) holds if and only if $\Theta_{J, T}^{*} u=0$ and $v=0$. Consequently,

$$
\mathbb{H}_{J, T}=\left(\mathcal{N}_{J} \otimes \mathcal{D}_{T}\right) \ominus \Theta_{J, T}\left(\mathcal{N}_{J} \otimes \mathcal{D}_{T^{*}}\right) .
$$

In this case, $\left.P_{\mathcal{N}_{J} \otimes \mathcal{D}_{T}}\right|_{\mathbb{H}_{J, T}}$ is the restriction operator, and relation (2.10) implies

$$
\mathbb{T}_{i}=P_{\mathbb{H}_{J, T}}\left(B_{i} \otimes I_{\mathcal{D}_{T}}\right) \mid \mathbb{H}_{J, T}, \quad i=1, \ldots, n .
$$


Conversely, if $\Theta_{J, T}$ is inner, then it is a partial isometry. Theorem 2.1 implies that $K_{J, T}$ is a partial isometry. Since $T$ is c.n.c., Corollary 1.2 implies

$$
\text { SOT- } \lim _{k \rightarrow \infty} \sum_{|\alpha|=k} T_{\alpha} T_{\alpha}^{*}=0,
$$

which proves that $T$ is a pure row contraction. This completes the proof.

As in the noncommutative case [12, one can easily prove the following.

Proposition 2.3. If $T:=\left[T_{1}, \ldots, T_{n}\right], T_{i} \in B(\mathcal{H})$, is a $J$-constrained c.n.c. row contraction, then $\Theta_{J, T}$ is outer if and only if there is no element $h \in \mathcal{H}, h \neq 0$, such that $\lim _{k \rightarrow \infty} \sum_{|\alpha|=k} T_{\alpha} T_{\alpha}^{*} h=0$.

Proof. Due to Corollary 1.2, the condition above is equivalent to $\operatorname{ker}\left(I-K_{J . T}^{*} K_{J, T}\right)$ $=\{0\}$. Using Theorem 2.1, we deduce that the latter equality is equivalent to

$$
\operatorname{ker} \Theta_{J, T} \Theta_{J, T}^{*}=\operatorname{ker}\left(I-K_{J . T} K_{J, T}^{*}\right)=\{0\},
$$

which is the same as $\Theta_{J, T}$ having dense range. The proof is complete.

Remark 2.4. If $J=\{0\}$ in Theorem 2.2 one can recover the model theorem for arbitrary c.n.c. row contractions [12].

Let $\Phi \in \mathcal{W}\left(W_{1}, \ldots, W_{n}\right) \bar{\otimes} B\left(\mathcal{K}_{1}, \mathcal{K}_{2}\right)$ and $\Phi^{\prime} \in \mathcal{W}\left(W_{1}, \ldots, W_{n}\right) \bar{\otimes} B\left(\mathcal{K}_{1}^{\prime}, \mathcal{K}_{2}^{\prime}\right)$ be two multi-analytic operators with respect to $B_{1}, \ldots, B_{n}$. We say that $\Phi$ and $\Phi^{\prime}$ coincide if there are two unitary operators $\tau_{j} \in B\left(\mathcal{K}_{j}, \mathcal{K}_{j}^{\prime}\right), j=1,2$, such that

$$
\Phi^{\prime}\left(I_{\mathcal{N}_{J}} \otimes \tau_{1}\right)=\left(I_{\mathcal{N}_{J}} \otimes \tau_{2}\right) \Phi .
$$

We remark that if $1 \in \mathcal{N}_{J}$, then the $C^{*}$-algebra $C^{*}\left(B_{1}, \ldots, B_{n}\right)$ is irreducible (see 22]). In this case, the operators $\Phi$ and $\Phi^{\prime}$ coincide if and only if there are two multi-analytic operators $U_{j}: \mathcal{N}_{J} \otimes \mathcal{K}_{j} \rightarrow \mathcal{N}_{J} \otimes \mathcal{K}_{j}^{\prime}$ such that $\Phi^{\prime} U_{1}=U_{2} \Phi$.

The next result shows that the constrained characteristic function is a complete unitary invariant for c.n.c. constrained row contractions.

Theorem 2.5. Let $J \neq F_{n}^{\infty}$ be a WOT-closed two-sided ideal of $F_{n}^{\infty}$ and let $T:=$ $\left[T_{1}, \ldots, T_{n}\right], T_{i} \in B(\mathcal{H})$, and $T^{\prime}:=\left[T_{1}^{\prime}, \ldots, T_{n}^{\prime}\right], T_{i}^{\prime} \in B\left(\mathcal{H}^{\prime}\right)$, be two $J$-constrained c.n.c. row contractions. Then $T$ and $T^{\prime}$ are unitarily equivalent if and only if their constrained characteristic functions $\Theta_{J, T}$ and $\Theta_{J, T^{\prime}}$ coincide.

Proof. Assume that $T$ and $T^{\prime}$ are unitarily equivalent and let $U: \mathcal{H} \rightarrow \mathcal{H}^{\prime}$ be a unitary operator such that $T_{i}=U^{*} T_{i}^{\prime} U$ for any $i=1, \ldots, n$. Simple computations reveal that

$$
U \Delta_{T}=\Delta_{T^{\prime}} U \quad \text { and } \quad\left(\bigoplus_{i=1}^{n} U\right) \Delta_{T^{*}}=\Delta_{T^{* *}}\left(\bigoplus_{i=1}^{n} U\right) .
$$

Define the unitary operators $\tau$ and $\tau^{\prime}$ by setting

$$
\tau:=U \mid \mathcal{D}_{T}: \mathcal{D}_{T} \rightarrow \mathcal{D}_{T^{\prime}} \quad \text { and } \quad \tau^{\prime}:=\left(\bigoplus_{i=1}^{n} U\right) \mid \mathcal{D}_{T^{*}}: \mathcal{D}_{T *} \rightarrow \mathcal{D}_{T^{\prime *}} .
$$

Taking into account the definition of the constrained characteristic function, it is easy to see that

$$
\left(I_{\mathcal{N}_{J}} \otimes \tau\right) \Theta_{J, T}=\Theta_{J, T^{\prime}}\left(I_{\mathcal{N}_{J}} \otimes \tau^{\prime}\right)
$$


Conversely, assume that the constrained characteristic functions of $T$ and $T^{\prime}$ coincide. Then there exist unitary operators $\tau: \mathcal{D}_{T} \rightarrow \mathcal{D}_{T^{\prime}}$ and $\tau_{*}: \mathcal{D}_{T^{*}} \rightarrow \mathcal{D}_{T^{*}}$ such that

$$
\left(I_{\mathcal{N}_{J}} \otimes \tau\right) \Theta_{J, T}=\Theta_{J, T^{\prime}}\left(I_{\mathcal{N}_{J}} \otimes \tau_{*}\right) .
$$

It is easy to see that (2.12) implies $\Delta_{J, T}=\left(I_{\mathcal{N}_{J}} \otimes \tau_{*}\right)^{*} \Delta_{J, T^{\prime}}\left(I_{\mathcal{N}_{J}} \otimes \tau_{*}\right)$ and

$$
\left(I_{\mathcal{N}_{J}} \otimes \tau_{*}\right) \overline{\Delta_{J, T}\left(\mathcal{N}_{J} \otimes \mathcal{D}_{T^{*}}\right)}=\overline{\Delta_{J, T^{\prime}}\left(\mathcal{N}_{J} \otimes \mathcal{D}_{T^{\prime *}}\right)} .
$$

Using the notations of Theorem 2.2 , we define the unitary operator $U: \mathbb{K}_{J, T} \rightarrow$ $\mathbb{K}_{J, T^{\prime}}$ by setting $U:=\left(I_{\mathcal{N}_{J}} \otimes \tau\right) \oplus\left(I_{\mathcal{N}_{J}} \otimes \tau_{*}\right)$. Straightforward computations reveal that the operator $\Phi: \mathcal{N}_{J} \otimes \mathcal{D}_{T^{*}} \rightarrow \mathbb{K}_{J, T}$, defined in the proof of Theorem 2.2, and the corresponding $\Phi^{\prime}$ satisfy the relations

$$
U \Phi\left(I_{\mathcal{N}_{J}} \otimes \tau_{*}\right)^{*}=\Phi^{\prime}
$$

and

$$
\left(I_{\mathcal{N}_{J}} \otimes \tau\right) P_{\mathcal{N}_{J} \otimes \mathcal{D}_{T}}^{\mathbb{K}_{J, T}} U^{*}=P_{\mathcal{N}_{J} \otimes \mathcal{D}_{T^{\prime}}}^{\mathbb{K}_{J, T^{\prime}}},
$$

where $P_{\mathcal{N}_{J} \otimes \mathcal{D}_{T}}^{\mathbb{K}_{J, T}}$ is the orthogonal projection of $\mathbb{K}_{J, T}$ onto $\mathcal{N}_{J} \otimes \mathcal{D}_{T}$. Notice that relation (2.13) implies

$$
\begin{aligned}
U \mathbb{H}_{J, T} & =U \mathbb{K}_{J, T} \ominus U \Phi\left(\mathcal{N}_{J} \otimes \mathcal{D}_{T^{*}}\right) \\
& =\mathbb{K}_{J, T^{\prime}} \ominus \Phi^{\prime}\left(I_{\mathcal{N}_{J}} \otimes \tau_{*}\right)\left(\mathcal{N}_{J} \otimes \mathcal{D}_{T^{*}}\right) \\
& =\mathbb{K}_{J, T^{\prime}} \ominus \Phi^{\prime}\left(\mathcal{N}_{J} \otimes \mathcal{D}_{T^{\prime *}}\right) .
\end{aligned}
$$

Therefore, the operator $\left.U\right|_{\mathbb{H}_{J, T}}: \mathbb{H}_{J, T} \rightarrow \mathbb{H}_{J, T^{\prime}}$ is unitary. On the other hand, we have

$$
\left(B_{i}^{*} \otimes I_{\mathcal{D}_{T^{\prime}}}\right)\left(I_{\mathcal{N}_{J}} \otimes \tau\right)=\left(I_{\mathcal{N}_{J}} \otimes \tau\right)\left(B_{i}^{*} \otimes I_{\mathcal{D}_{T}}\right) .
$$

Now, let $\mathbb{T}:=\left[\mathbb{T}_{1}, \ldots \mathbb{T}_{n}\right]$ and $\mathbb{T}^{\prime}:=\left[\mathbb{T}_{1}^{\prime}, \ldots \mathbb{T}_{n}^{\prime}\right]$ be the models provided by Theorem 2.2 for the row contractions $T$ and $T^{\prime}$, respectively. Using the relation (2.10) for $T^{\prime}$ and $T$, as well as relations (2.14) and (2.15), we deduce that

$$
\begin{aligned}
P_{\mathcal{N}_{J} \otimes \mathcal{D}_{T^{\prime}}}^{\mathbb{K}_{J, T^{\prime}}} \mathbb{T}_{i}^{\prime *} U x & =\left(B_{i}^{*} \otimes I_{\mathcal{D}_{T^{\prime}}}\right) P_{\mathcal{N}_{J} \otimes \mathcal{D}_{T}}^{\mathbb{K}_{J, T}} U x=\left(B_{i}^{*} \otimes I_{\mathcal{D}_{T^{\prime}}}\right)\left(I_{\mathcal{N}_{J}} \otimes \tau\right) P_{\mathcal{N}_{J} \otimes \mathcal{D}_{T}}^{\mathbb{K}_{J, T}} x \\
& =\left(I_{\mathcal{N}_{J}} \otimes \tau\right)\left(B_{i}^{*} \otimes I_{\mathcal{D}_{T}}\right) P_{\mathcal{N}_{J} \otimes \mathcal{D}_{T}}^{\mathbb{K}_{J, T}} x=\left(I_{\mathcal{N}_{J}} \otimes \tau\right) P_{\mathcal{N}_{J} \otimes \mathcal{D}_{T}}^{\mathbb{K}_{J}} T_{i}^{*} x \\
& =P_{\mathcal{N}_{J} \otimes \mathcal{D}_{T^{\prime}}}^{\mathbb{K}_{J, T}} U \mathbb{T}_{i}^{*} x
\end{aligned}
$$

for any $x \in \mathbb{H}_{J, T}$ and $i=1, \ldots, n$. Consequently, since $\left.P_{\mathcal{N}_{J} \otimes \mathcal{D}_{T^{\prime}}}^{\mathbb{K}_{J T^{\prime}}}\right|_{\mathbb{H}_{J, T^{\prime}}}$ is a one-toone operator (see relation (2.9)), we obtain

$$
\left(\left.U\right|_{\mathbb{H}_{J, T}}\right) \mathbb{T}_{i}^{*}=\mathbb{T}_{i}^{\prime *}\left(\left.U\right|_{\mathbb{H}_{J, T}}\right), \quad i=1, \ldots, n .
$$

Now, using Theorem 2.2, we conclude that $T$ and $T^{\prime}$ are unitarily equivalent. The proof is complete.

As in the noncommutative case [19, one can prove the following.

Proposition 2.6. Let $J \neq F_{n}^{\infty}$ be a WOT-closed two-sided ideal of $F_{n}^{\infty}$ such that $1 \in \mathcal{N}_{J}$. If $T:=\left[T_{1}, \ldots, T_{n}\right]$ is a $J$-constrained c.n.c. row contraction, then $T$ is unitarily equivalent to a constrained shift $\left[B_{1} \otimes I_{\mathcal{K}}, \ldots, B_{n} \otimes I_{\mathcal{K}}\right]$ for some Hilbert space $\mathcal{K}$ if and only if $\Theta_{J, T}=0$. 
Proof. If $T=\left[B_{1} \otimes I_{\mathcal{K}}, \ldots, B_{n} \otimes I_{\mathcal{K}}\right]$, then $K_{J, T} f=f$ for $f \in \mathcal{N}_{J} \otimes \mathcal{K}$. Indeed, since $1 \in \mathcal{N}_{J}$ for any $f=\sum_{\alpha \in \mathbb{F}_{n}^{+}} e_{\alpha} \otimes k_{\alpha}$ in $N_{J} \otimes \mathcal{K} \subseteq F^{2}\left(H_{n}\right) \otimes \mathcal{K}$, we have

$$
\begin{aligned}
K_{J, T} f & =\sum_{\alpha \in \mathbb{F}_{n}^{+}} P_{\mathcal{N}_{J}} e_{\alpha} \otimes P_{\mathcal{K}}\left(B_{\alpha}^{*} \otimes I_{\mathcal{K}}\right) f=\sum_{\alpha \in \mathbb{F}_{n}^{+}} P_{\mathcal{N}_{J}} e_{\alpha} \otimes P_{\mathcal{K}}\left(S_{\alpha}^{*} \otimes I_{\mathcal{K}}\right) f \\
& =\sum_{\alpha \in \mathbb{F}_{n}^{+}} P_{\mathcal{N}_{J}} e_{\alpha} \otimes k_{\alpha}=P_{\mathcal{N}_{J} \otimes \mathcal{K}} f=f .
\end{aligned}
$$

Now, Theorem 2.1 shows that $\Theta_{J, T}=0$. Conversely, if $\Theta_{J, T}=0$, then Theorem 2.2 shows that $T$ is unitarily equivalent to the constrained shift $\left[B_{1} \otimes I_{\mathcal{D}_{T}}, \ldots, B_{n} \otimes\right.$ $\left.I_{\mathcal{D}_{T}}\right]$.

Remark 2.7. All the results of this section can be written in the particular case when $T:=\left[T_{1}, \ldots, T_{n}\right]$ is a $q$-commuting c.n.c. row contraction, i.e.,

$$
T_{i} T_{j}=q_{j i} T_{j} T_{i}, \quad 1 \leq i<j \leq n,
$$

where $q_{i j} \in \mathbb{C}$.

Notice that $T$ is $q$-commuting if and only if it is a $J_{q}$-constrained row contraction, where $J_{q}$ is the WOT-closed two-sided ideal of $F_{n}^{\infty}$ generated by the $q$-commutators $S_{i} S_{j}-q_{j i} S_{j} S_{i}, 1 \leq i<j \leq n$, where $S_{1}, \ldots, S_{n}$ are the left creation operators on the full Fock space. We refer to 5 , for related results on $q$-commuting row contractions.

We remark that in the particular case when $q_{i j}=1$ we obtain a model theory for commuting c.n.c. row contractions.

\section{REFERENCES}

[1] J. Arazy and M. Englis̆, Analytic models for commuting operator tuples on bounded symmetric domains, Trans. Amer. Math. Soc. 355 (2003), no.2, 837-864. MR1932728 (2003k:47019)

[2] A. Arias and G. Popescu, Noncommutative interpolation and Poisson transforms, Israel J. Math. 115 (2000), 205-234. MR.1749679 (2001i:47021)

[3] W.B. Arveson, Subalgebras of $C^{*}$-algebras III: Multivariable operator theory, Acta Math. 181 (1998), 159-228. MR.1668582 (2000e:47013)

[4] C. Benhida, And D. Timotin, Characteristic functions for multicontractions and automorphisms of the unit ball, preprint.

[5] B.V.R. Bhat and T. Bhattacharyya, A model theory for $q$-commuting contractive tuples, J. Operator Theory 47 (2002), 1551-1568. MR1905815 (2003c:47018)

[6] T. Bhattacharyya, J. Eschmeier, and J. Sarkar, Characteristic function of a pure commuting contractive tuple, Integral Equation Operator Theory, 53 (2005), 23-32. MR2183594

[7] T. Bhattacharyya, J. Eschmeier, and J. Sarkar, On commuting c.n.c. contractive tuples, preprint.

[8] J.W. Bunce, Models for n-tuples of noncommuting operators, J. Funct. Anal. 57 (1984), 21-30. MR 0744917 (85k:47019)

[9] A. E. Frazho, Models for noncommuting operators, J. Funct. Anal. 48 (1982), no.1, 1-11. MR0671311 (84h:47010)

[10] G. POPESCU, Models for infinite sequences of noncommuting operators, Acta. Sci. Math. (Szeged) 53 (1989), 355-368. MR1033608 (91b:47025)

[11] G. Popescu, Isometric dilations for infinite sequences of noncommuting operators, Trans. Amer. Math. Soc. 316 (1989), 523-536. MR0972704 (90c:47006)

[12] G. Popescu, Characteristic functions for infinite sequences of noncommuting operators, $J$. Operator Theory 22 (1989), 51-71. MR.1026074 (91m:47012)

[13] G. Popescu, Multi-analytic operators and some factorization theorems, Indiana Univ. Math. J. 38 (1989), 693-710. MR.1017331 (90k:47019) 
[14] G. Popescu, Von Neumann inequality for $\left(B(H)^{n}\right)_{1}$, Math. Scand. 68 (1991), 292-304. MR:1129595 (92k:47073)

[15] G. POPESCU, Functional calculus for noncommuting operators, Michigan Math. J. 42 (1995), 345-356. MR1342494 (96k:47025)

[16] G. Popescu, Multi-analytic operators on Fock spaces, Math. Ann. 303 (1995), 31-46. MR1348353 (96k:47049)

[17] G. Popescu, Poisson transforms on some $C^{*}$-algebras generated by isometries, J. Funct. Anal. 161 (1999), 27-61. MR 1670202 (2000m:46117)

[18] G. Popescu, Commutant lifting, tensor algebras, and functional calculus, Proc. Edinb. Math. Soc. 44 (2001), 389-406. MR1880399 (2003f:47125)

[19] G. Popescu, Curvature invariant for Hilbert modules over free semigroup algebras, Adv. Math. 158 (2001), 264-309. MR.1822685 (2002b:46097)

[20] G. Popescu, Central intertwining lifting, suboptimization, and interpolation in several variables, J. Funct. Anal. 189 (2002), 132-154. MR.1887631 (2002m:47013)

[21] G. Popescu, Unitary invariants in multivariable operator theory, preprint 2004.

[22] G. Popescu, Operator theory on noncommutative varieties, Indiana Univ. Math. J. 56 (2006), No.2, 389-442.

[23] B. Sz.-Nagy And C. FoIAs,, Harmonic Analysis of Operators on Hilbert Space, NorthHolland, New York 1970. MR0275190 (43:947)

[24] J. von Neumann, Eine Spectraltheorie für allgemeine Operatoren eines unitären Raumes, Math. Nachr. 4 (1951), 258-281. MR0043386(13:254a)

Department of Mathematics, The University of Texas at San Antonio, San Antonio, TEXas 78249

E-mail address: gelu.popescu@utsa.edu 